\title{
Traduire
}

Revue française de la traduction

227 | 2012

Éco, socio, philo... \& co

\section{Être traducteur économique et financier aujourd'hui : défis et avantages}

\section{Verónica Román}

Traducteur : Aurélie Barbe

URL : http://journals.openedition.org/traduire/481

DOI : 10.4000/traduire.481

ISSN : 2272-9992

\section{Éditeur}

Société française des traducteurs

\section{Édition imprimée}

Date de publication : 15 décembre 2012

Pagination : 62-76

ISSN : 0395-773X

Référence électronique

Verónica Román, « Être traducteur économique et financier aujourd'hui : défis et avantages », Traduire [En ligne], 227 | 2012, mis en ligne le 01 décembre 2014, consulté le 19 avril 2019. URL : http:// journals.openedition.org/traduire/481 ; DOI : 10.4000/traduire.481 


\title{
Être traducteur économique et financier aujourd'hui : défis et avantages
}

\author{
Verónica Román
}

Rares sont les études consacrées au marché professionnel de la traduction et celles portant sur la traduction dans le domaine des affaires et, plus concrètement, dans le secteur économique et financier font figure d'exception.

\section{La traduction, un marché en pleine croissance}

Le marché de la traduction se porte bien. En rapide évolution depuis une vingtaine d'années, et avec une croissance annuelle de 5 à 7,5\% entre 2005 et 2010, il n'est même pas près de s'essouffler, selon une étude menée à son sujet en Europe par l'EUATC (European Union of Associations of Translation Companies) en 2005. Dans son article paru dans la revue électronique allemande Tcworld (revue sur la gestion de l'information par les entreprises en cours d'internationalisation) portant sur les prévisions de croissance du marché de la traduction dans deux décennies à venir, Hager (2008) prévoyait une forte augmentation des volumes pendant cette période. Selon Common Sense Advisory, Inc.(1), cabinet de conseil américain, le marché professionnel de la traduction et de l'interprétation ne cesse de se développer, et ce, malgré la récession économique mondiale et un certain ralentissement de la croissance en 2010.

Aux États-Unis, une pluralité linguistique de plus en plus poussée ne pourra se solder que par une demande accrue de prestations de traduction. Par ailleurs, le forum About Translation forum consacré à la traduction professionnelle - cite un article publié en 2006 par le New York Times mettant en avant l'importance de la croissance constatée dans le secteur de la traduction et de l'interprétation. L'article prévoyait une hausse de $20 \%$ du nombre des traducteurs et interprètes entre 2004 et 2014 d'après les chiffres de l'Institut fédéral des statistiques aux États-Unis.

(1) Common Sense Advisory, 2010. 


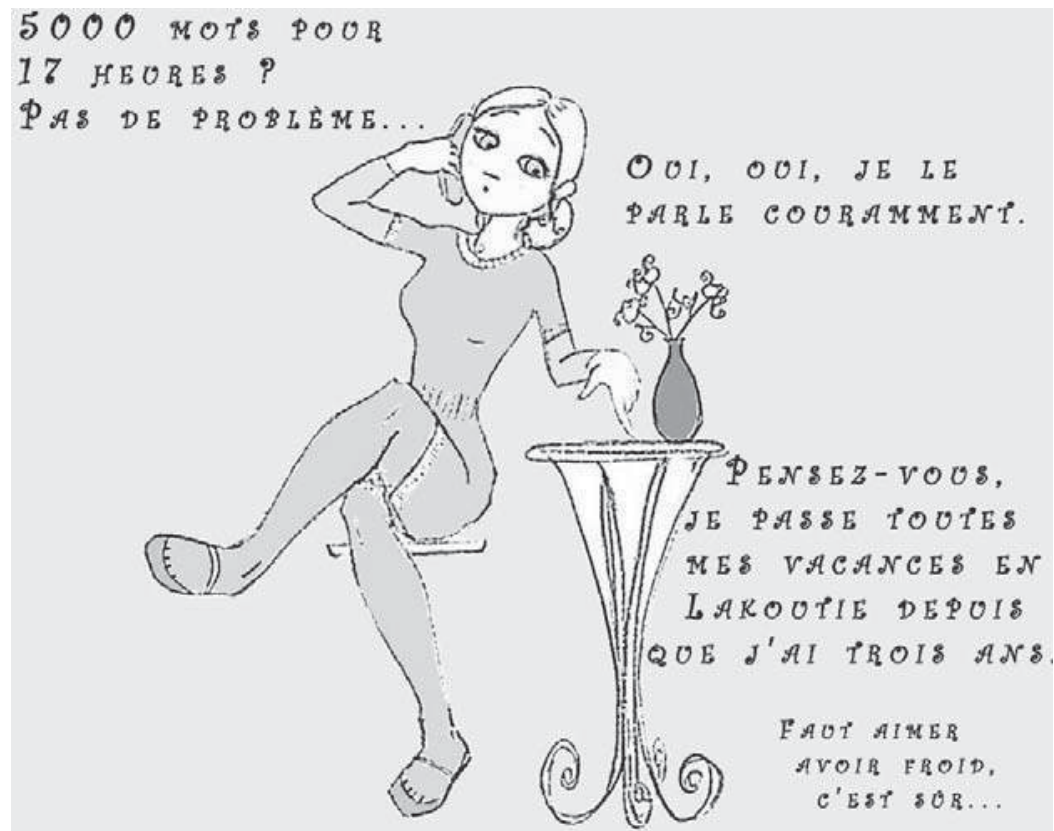

Illustration : Marlène Junius, http://alotoftralala.over-blog.com

Depuis l'ouverture de l'Espagne vers l'extérieur en 2010, les exportations ont connu un bond de 17,4 \%, selon les données publiées par le ministère de l'Industrie, du Tourisme et du Commerce, le pays figurant en 2011 - selon les prévisions de l'OCDE, reprises dans l'étude de l'Institut des études économiques (IEE) - parmi ceux dont les exportations ont le plus fortement augmenté à travers le monde. De surcroît, l'OCDE place l'Espagne à la cinquième place en termes de croissance du commerce extérieur, en tablant sur une hausse de 9,9\% des exportations de biens et services en 2011.

L'ouverture des marchés et l'internationalisation provoquent une hausse de la demande de traductions de la part de nombreux organismes et entreprises. Selon une étude réalisée par I'Asociación Española de Empresas de Traducción (ACT) sur le marché de la traduction en Espagne, la croissance annuelle moyenne dans le secteur s'élevait à $6 \%$ en 2004(2).

Comme le souligne un article publié dans Mercado de Trabajo(3), les perspectives du marché de la traduction en Espagne sont très encourageantes (le secteur connaissant des taux de

(2) ACT, 2004.

(3) Mercado de Trabajo, 2007. 
croissance annuelle dans la fourchette de 6 à $8 \%$ ), la suppression de barrières linguistiques représentant l'une des étapes clés pour toute entreprise souhaitant consolider son développement international. Selon Mme Celia Rico, professeure de traduction à l'Universidad Europea de Madrid, dans un article portant sur les PME et entreprises assimilées publié sur le portail de Microsoft(4), un pourcentage non négligeable d'entreprises perdrait des marchés à l'exportation faute de compétences linguistiques et culturelles, entrainnant ainsi des manques à gagner d'une valeur parfois supérieure au million d'euros.

\section{Qui dit globalisation, dit grands besoins en matière de traduction économique et financière}

Dans le monde globalisé d'aujourd'hui, où les distances dans le temps et dans l'espace ne cessent de décroître, les besoins des entreprises dépassent, eux aussi, les frontières. Prenons, par exemple, l'exportation de biens et de services d'un pays à l'autre ou alors les innombrables flux économiques et financiers qui existent de nos jours entre entreprises, pays ou organisations internationales. Ces opérations commerciales et cette ouverture des sociétés sur le monde multiplient les besoins en matière de traduction économique et financière, ce qui en fait l'une des spécialités les plus demandées sur le marché et explique l'essor des débouchés pour les traducteurs exerçant leur métier dans ce domaine. La plupart du temps, les entreprises à vocation internationale externalisent la traduction de leurs gros volumes documentaires.

\section{La crise financière ou comment coordonner les restrictions budgétaires en matière de traduction avec la nécessité de traductions économiques et financières de qualité}

Depuis 2007, le monde traverse une crise financière d'ampleur internationale qui a commencé par la vente des titres subprime baptisés "valeurs poubelles " aux États-Unis. Suivie par la faillite, quelques mois plus tard, de Lehman Brothers, quatrième banque d'investissement aux États-Unis, cette crise s'est prolongée jusqu'à aujourd'hui avec celle de la dette souveraine en Europe. À l'instar des autres pays européens, l'Espagne présente un taux de croissance économique très faible. Tant il est vrai qu'en période de crise, les entreprises ont tendance à mettre en place des limitations budgétaires en matière de traduction, il va néanmoins sans dire que, dans le cas d'enjeux financiers importants - par exemple, lors d'opérations économiques et financières - il est indispensable de faire appel à des traducteurs indépendants de qualité pour surmonter les barrières linguistiques capables de faire échouer les transactions en question. S'adressant aux étudiants lors d'un séminaire sur le marché professionnel de la

(4) Microsoft, 2011. 
traduction économique et financière organisé par l'auteure de cet article à l'Universidad Autonoma de Madrid, Javier Gil(5) a souligné l'importance des traducteurs économiques et financiers : "Quand il y va de son argent, tout le monde veut examiner le texte dans le détail et ceci dans sa langue maternelle. "

\section{Le marché de la traduction : un marché désorganisé, non réglementé et concurrentiel}

Dans son rapport sur le marché de la traduction professionnelle en Europe, l'EUATC(6) relève que la traduction est l'un des marchés les plus fragmentés qui soient. Fortement marqué par la concurrence, il est peu structuré et totalement désuni. Comme le signale cette association, même si les traducteurs indépendants et les agences de traduction partagent certains intérêts communs, il n'existe aucune communication entre eux, ce qui donne au secteur une image très négative. De plus, tous ceux qui connaissent tant soit peu le marché de la traduction savent qu'il est dépourvu de tout cadre règlementaire. La profession est ouverte à toutes et à tous, n'importe qui peut y accéder : pour exercer le métier de traducteur, il suffit d'être équipé d'un ordinateur et d'un petit espace de travail (la grande majorité des traducteurs indépendants travaillant chez eux).

Dans l'ensemble, nous pouvons affirmer que l'offre en matière de traduction est bien supérieure à la demande. Nous entendons par offre celle constituée aussi bien par les agences de traduction et les traducteurs indépendants que par les services de traduction des multi-nationales ou des organismes publics. Selon l'EUATC(7), les agences de traduction représentent $20 \%$ du marché et les traducteurs indépendants $80 \%$. En Espagne, de nombreuses universités proposent actuellement des cursus en traduction et interprétation. Le nombre de traducteurs se lançant sur le marché du travail ne cesse donc de s'accroître d'année en année. À cela, il faut ajouter le grand nombre de diplômés en langues ou encore en droit, en économie ou en gestion d'entreprises, entre autres, qui exercent notre métier. Et dans le cas précis de l'espagnol, l'offre de traducteurs est encore bien supérieure si l'on tient compte des nombreux pays hispanophones.

Conséquence de cet excédent de traducteurs professionnels : la multiplication des agences de traduction (dont le nombre a considérablement augmenté ces dernières années) et des traducteurs indépendants qui, pour attirer le client, pratiquent des tarifs très bas et créent ainsi une concurrence déloyale sur le web. Sans compter les nombreux jeunes diplômés qui cassent les prix afin de se tailler une place sur le marché. Comme le signalent plusieurs auteurs

(5) Directeur de l'entreprise Equality Financial Translations.

(6) EUATC, 2005.

(7) EUATC, 2005 
dans une étude réalisée sur les débouchés professionnels dans la traduction médicale(8), l'écart entre les prix pratiqués dans le monde hispanophone et ceux pratiqués dans l'ensemble de l'Europe ou aux États-Unis est scandaleux. D'ailleurs, on pourrait tirer la même conclusion pour le secteur économique et financier. En Espagne, les particuliers et les entreprises publiques ou privées demandeurs de services de traduction à des tarifs réduits ne manquent pas.

Parallèlement, certaines agences de traduction, qui jouent le rôle d'intermédiaire sur le marché et qui exploitent cette position pour sauvegarder leur compétitivité aux dépens du traducteur indépendant, proposent à ce dernier des tarifs de misère, sachant qu'elles trouveront toujours preneur. Force est d'ailleurs de constater que la législation sur la défense de la concurrence ne comporte aucune règle établie sur les conditions de travail et les tarifs convenus entre les professionnels du secteur et les clients. L'offre étant supérieure à la demande, le client se retrouve parfois avec un produit final qui ne correspond pas forcément à ses attentes ou bien avec une traduction de qualité médiocre. Malheureusement, c'est souvent le critère du prix qui l'emporte sur celui de la qualité. De plus, la crise économique actuelle déjà citée pousse bon nombre de traducteurs à baisser leurs tarifs dans l'espoir de rester concurrentiels et de continuer à s'attirer des clients. Bien sûr, la plupart des associations du secteur dénoncent ces pratiques, cette tendance à " tirer les prix vers le bas ", à " casser le marché ", en insistant sur la nécessité de respecter des prix plancher en matière de prestation de services de traduction.

L'EUATC(9) signale, en outre, que la traduction - considérée comme une dépense ou un bien de consommation et non comme un investissement - renvoie une image plutôt négative. De même, l'expérience nous montre la totale méconnaissance de la difficulté et de la complexité du travail de traducteur. Arevalillo Duval(10) constate aussi l'existence d'une grande ignorance de la traduction et de ses processus chez les autres professionnels. Tout au long de ma carrière, j'ai pu constater qu'en Espagne, nombreux sont ceux qui croient que la seule connaissance de deux langues peut faire d'eux un traducteur. Forts de cette conviction, certains clients s'attendent à ce que le travail soit exécuté dans des délais très serrés et à moindre coût, quelles que soient la nature et la complexité du texte. La rapidité, surtout dans le milieu des affaires, joue un rôle primordial dans le choix d'un prestataire de services de traduction. Malheureusement, au cours de ma carrière, j'ai souvent été sollicitée par des agences ou par des particuliers pour traduire des documents économiques et financiers volumineux d'un niveau de difficulté élevé sous un délai de 24 à 48 heures. Autre exemple vécu, relatif cette fois aux traductions économiques et financières assermentées : le client envoie un texte traduit par ses propres soins ou par une autre personne ayant quelques connaissances de la langue source dans l'espoir d'en obtenir rapidement une version assermentée. Le texte étant déjà traduit, il suffit aux yeux du client d'y " apposer le cachet officiel "...

(8) Mayor Serrano, Arevalillo, Castro Roig, De Miguel y Turrión, 2004.

(9) EUATC, 2005

(10) Arevalillo Duval, 2006b.

Traduire $\mathrm{n}^{\circ} 227$, décembre 2012 
Face à ce tableau - secteur non réglementé, offre excessive et fragmentée, méconnaissance des difficultés du métier, baisse des prix et concurrence déloyale -, la norme européenne de qualité EN 15038 (UNE-EN 15038 dans sa version espagnole officielle) approuvée à l'initiative de l'EUATC prévoit, jusqu'à nouvel ordre, une standardisation en matière de services de traduction. Cette norme vise à définir les critères de qualité attendus de la part des prestataires de services de traduction. À cette fin, elle prévoit (§ 5.2 ) la révision de la traduction par le traducteur lui-même, ainsi que par un tiers (réviseur) et par un autre professionnel (spécialiste du sujet). II va sans dire que cette norme ne pourra jamais régler tous les maux du secteur, mais elle a le mérite d'établir des lignes directrices et de fournir un cadre de référence pour l'exercice de notre profession.

\section{Le marché de la traduction économique et financier : un marché hétérogène et dynamique, mais enrichissant pour le traducteur}

Si nous partons du constat déjà posé que le marché de la traduction est un marché compétitif, nous devrons alors nous demander quelles sont les particularités de la traduction de textes économiques et financiers qui en font un domaine moins concurrencé que d'autres. Pourquoi le nombre de traducteurs qui se positionnent sur ce créneau est-il moins important et pourquoi ce type de traduction s'avère-t-il plus rentable ?

Tout d'abord, le marché professionnel de la traduction économique et financière est hétérogène et dynamique. Cette hétérogénéité s'explique non seulement par le grand nombre de clients susceptibles de commander des traductions dans ce domaine, mais aussi par leur diversité (agences de traduction, entreprises de conseil, banques commerciales ou d'investissement, institutions financières, cabinets d'avocats à dimension internationale, organismes publics, particuliers, etc.). Chacun d'entre eux exerçant des métiers très variés, leurs besoins en matière de traduction diffèrent de l'un à l'autre. Des années d'expérience nous permettent d'affirmer qu'un traducteur économique et financier ne reçoit jamais deux commandes identiques. En effet, même si, au départ, certains textes sources peuvent paraître similaires, les clients demandeurs sont différents - chacun ayant une structure et un modèle organisationnel propres - ce qui conditionne fortement le produit fini offert par le professionnel. Par exemple, dans le secteur privé, en fonction de la structure et de l'organisation de chaque entreprise, les demandes de traductions économiques et financières émanent aussi bien des services de communication, de conseil juridique, de relations investisseurs que des secrétariats de conseillers délégués, de présidents ou de services commerciaux. N'oublions pas qu'un grand nombre de décisions relatives à la traduction - par exemple, les choix terminologiques - dépendent du client. En outre, parmi les autres facteurs qui influencent la qualité de la traduction, citons le format du document, les outils informatiques exigés par le client, la lisibilité ou la clarté du texte source, les tarifs proposés ou le délai exigé. Les connaissances pratiques et l'expérience professionnelle du traducteur peuvent s'avérer une aide précieuse lorsqu'il doit 
faire face aux flots d'exigences imposées par le client. Sans compter que ce dernier n'est pas forcément le destinataire de la traduction! Bien souvent, le traducteur ignore à qui se destine le texte cible qu'il est en train d'élaborer, ce qui rend ses choix encore plus délicats.

Si le cœur de métier de chaque client est très varié et très hétérogène, le travail du traducteur - bien que difficile - s'en trouve d'autant plus passionnant et enrichissant. Afin de mieux comprendre le discours de son client ainsi que la terminologie et la phraséologie employées, le traducteur doit connaître, dans ses grandes lignes, la nature de l'activité de l'entreprise pour laquelle il traduit. Compte tenu de la diversité et du nombre de demandeurs de traduction dans le secteur économique et financier - sans oublier le nombre de situations nécessitant l'intervention d'un traducteur - on peut affirmer que le traducteur ayant pour spécialité cette discipline touche nécessairement à différents sous-domaines, qu'il doit être prêt à se documenter sur les entreprises pour lesquelles il traduit et qu'il doit savoir se conformer à leurs différentes exigences ou à leurs différents besoins dans chaque cas de figure.

L'hétérogénéité de son travail - grande source d'enrichissement pour lui en tant que traducteur économique et financier - lui permet d'échapper à la monotonie. II suffit de lire les rubriques Économie et Finance des journaux nationaux et internationaux pour se rendre compte du dynamisme du monde économique et - surtout - financier d'aujourd'hui, le secteur est constamment en mouvement et en ébullition perpétuelle. Du jour au lendemain, des entreprises se retrouvent en cessation de paiement, fusionnent, sont rachetées, disparaissent, les banques et les États font faillite, l'euro est menacé. Nous traversons une nouvelle époque, une période de turbulence où les changements se suivent et se chevauchent. C'est pourquoi le traducteur doit faire preuve de professionnalisme, de flexibilité et de polyvalence, être capable de s'adapter aux évolutions économiques et financières, rester au courant des nouveautés terminologiques - conséquences de ces évolutions - et pouvoir assimiler les innovations technologiques, c'est-à-dire les outils de traduction (logiciels de gestion terminologique, mémoires de traduction, traduction assistée) et les ressources documentaires lui permettant de combler les lacunes thématiques, conceptuelles et terminologiques qui le guettent dans son travail.

\section{La traduction économique et financière, une tâche complexe et difficile : un défi pour le traducteur indépendant}

Autre caractéristique du marché de la traduction économique et financière : sa grande complexité et les énormes difficultés intrinsèques de la tâche. Cette complexité explique également la relative faiblesse de la concurrence sur ce créneau et la supériorité des tarifs qui s'y pratiquent. Elle découle à mes yeux :

- de la complexité même du monde actuel, du grand nombre d'interrelations existant entre les pays et des multiples flux commerciaux entre les multinationales, celles-ci étant devenues des acteurs sociaux de premier plan. 
Être traducteur économique et financier aujourd'hui : défis et avantages

- de l'étendue des connaissances que demande la traduction de textes économiques et financiers. Comme de précédentes études l'ont démontré, dans de nombreux documents professionnels du monde des affaires, la frontière entre les différents vocabulaires spécialisés est assez floue. Les textes dans lesquels le jargon juridique cohabite avec le langage économique et financier sont légion. Quelques exemples : plans d'options pour actions, conventions entre actionnaires, contrat d'achat-vente d'actions, actes et ordres du jour d'organes d'entreprise, etc. Ce constat entraîne une plus grande exigence de formation pour le traducteur. S'il est impossible pour le traducteur de dominer tous les thèmes susceptibles de figurer dans les textes, il devra néanmoins effectuer des recherches documentaires approfondies pour acquérir les connaissances techniques de base et comprendre les différents termes et expressions qu'il risque de trouver dans le texte source, s'il veut être en mesure de faire les meilleurs choix terminologiques.

- des délais serrés imposés par les clients dans cette spécialité (la majorité des besoins des entreprises sont urgents) et de la difficulté pour les traducteurs d'offrir un service de qualité en un laps de temps aussi réduit. C'est pourquoi le traducteur professionnel doit être en mesure de déterminer s'il est capable - ou non - de respecter le délai exigé. De prime abord, calculer un délai peut paraître d'une simplicité enfantine. Néanmoins, il n'est pas toujours évident d'étudier, dans les quelques minutes que lui laisse parfois le client, plusieurs variables, tels que la nature de l'entreprise et de ses activités, le type de texte et sa difficulté, les outils informatiques imposés par le client, ses exigences terminologiques, etc. Si le traducteur ne maîtrise pas comme il se doit le sous-domaine de spécialité du texte ou si ces différentes variables risquent de compliquer ou rendre impossible le respect des délais impartis, il est préférable de refuser la commande. Ainsi, le professionnel ne ternira pas sa réputation et pourra plus facilement gagner la confiance de son client. Les délais impartis impliquent que le traducteur doit être capable de travailler efficacement sous pression (le traducteur salarié bénéficie souvent de plages temporelles un peu plus larges que son homologue indépendant, qui doit savoir bien s'organiser pour se conformer aux délais).

Comme le signale Ricard Lozano dans son Vademécum del traductor, un traducteur digne de ce nom se doit de maximiser sa rentabilité, la productivité étant d'une importance primordiale à cet égard. II faut donc identifier tous les éléments favorisant la productivité pour jeter les bases d'une véritable rentabilité. À ce sujet, l'association ASETRAD (Asociación Española de Traductores Correctores e Intérpretes) dispose d'un outil appelé CalPro grâce auquel les traducteurs - considérés comme des microentreprises - peuvent vérifier combien ils gagnent, combien ils devraient gagner et estimer le nombre d'heures qu'ils devraient travailler afin d'atteindre leurs objectifs.

Cette complexité inhérente à la traduction économique et financière place les professionnels, pour qui formation permanente et travail doivent aller de pair, face à leurs responsabilités en matière de formation continue. lls doivent se former sans arrêt, c'est-à-dire tout au long de leur 
carrière, pour pouvoir proposer une offre de qualité et se distinguer de leurs concurrents directs. Leur spécialité leur permettra d'offrir une valeur ajoutée. De plus, la formation doit être vaste et multidisciplinaire, tout en englobant les différents aspects du métier. Le professionnel doit se former par rapport aux activités de chacun de ses clients, à la terminologie relative aux différents domaines liés à cette spécialité, aux outils informatiques ainsi qu'aux différents aspects de l'activité professionnelle, et ce, par le biais d'expériences en entreprise, par la participation à des cours de troisième cycle ou par l'autoformation, etc. Avec de tels bagages, le traducteur professionnel pourra offrir des services de qualité et imposer des tarifs plus élevés. Pour nous, le traducteur doit se fixer l'objectif de poursuivre son évolution - professionnellement parlant -, en vue de satisfaire ses clients et d'être reconnu pour son travail, tant d'un point de vue personnel que professionnel.

En ce qui concerne la formation des jeunes diplômés, il faut savoir qu'en Espagne l'offre de cours de troisième cycle dans le domaine de la traduction économique et financière est de très faible envergure. À l'heure actuelle, les programmes de traduction juridique de certaines universités (plus nombreux que les programmes de traduction économique et financière, ces derniers pouvant se compter sur les doigts d'une main) ne proposent pas de cours dans cette discipline ou, le cas échéant, les matières proposées se limitent souvent à la traduction dans le domaine commercial et bancaire. II paraît donc essentiel de mettre en place des programmes de formation spécialisés en traduction économique et juridique ou en traduction économique et financière.

Le marché de la traduction économique et financière est donc très exigeant et - répétonsle - le traducteur doit faire preuve de connaissances thématiques ou encyclopédiques (non seulement dans le domaine économique et financier, mais aussi dans les différentes disciplines afférentes à ce type de textes), de compétences documentaires lui permettant d'élargir le champ de ses connaissances, d'une certaine maîtrise des outils informatiques - source de compétitivité et d'efficacité en cas de délais réduits - ainsi que des compétences professionnelles intrinsèques à son métier. Concernant ces dernières, on se limitera au cas du traducteur indépendant, car il s'agit du mode d'exercice le plus répandu sur le marché. Quelles sont donc les qualités dont doit disposer le traducteur indépendant dans les domaines économique et financier? Elles pourraient se résumer comme suit :

- le traducteur doit savoir s'organiser afin de bien gérer son temps, cet aspect étant d'autant plus important dans le monde des affaires ;

- le traducteur doit savoir élaborer une stratégie de marketing. Avant même de s'installer à son compte, il doit définir les services qu'il souhaite offrir, les types de clients avec lesquels il a envie de travailler et les tarifs qu'il compte appliquer. Ensuite, il doit connaître les critères utilisés par les clients dans leur choix de prestataire, le budget qu'ils y consacrent, leurs besoins et comment les satisfaire, leurs secteurs d'activité, leur zone d'implantation 
Être traducteur économique et financier aujourd'hui : défis et avantages

géographique. Une fois que le traducteur a bien ciblé ses prospects, il doit mettre en application sa stratégie marketing et publicitaire afin de se constituer un bon portefeuille clients. II doit donc soigner sa visibilité sur le marché et bien préparer le terrain, en faisant, par exemple, appel à des méthodes traditionnelles - bouche à oreille, remise de cartes de visite, inscription dans les annuaires téléphoniques sous les rubriques "traduction " - ou à des méthodes plus technologiques telles que l'Internet via les divers forums, portails, moteurs de recherche, réseaux sociaux, etc. II est donc fortement recommandé au traducteur de disposer d'une page web professionnelle (l'une des méthodes de promotion les plus efficaces, car cela revient à laisser ouvert son bureau 24h/24, 7j/7, 365 jours par an(11)) avec son propre nom de domaine. Si le traducteur envoie son CV à des prospects, ce dernier doit être structuré, bien rédigé et, évidemment, centré sur son expérience dans le domaine de la traduction. II est également très important pour le traducteur d'assister à des congrès, à des séminaires, à des cours de formation ainsi qu'à toute autre activité en rapport avec la profession, tant pour se former que pour nouer des contacts professionnels ;

- le traducteur doit faire preuve d'aptitudes commerciales qui lui permettront de se forger une clientèle. Cet aspect est fortement lié à la stratégie marketing esquissée ci-dessus. II ne faut pas oublier que le traducteur - au-delà de traduire - doit vendre son produit. Au début de son activité professionnelle, comme tout commercial, le traducteur doit persévérer et s'armer de patience avant de voir ses efforts porter leurs fruits. De même, le professionnel de la traduction qui présente ses services à un prospect doit promettre une offre de qualité et proposer une certaine valeur ajoutée qui le distingue de ses concurrents ;

- le traducteur doit savoir gérer, organiser et planifier convenablement son travail, tenir sa comptabilité (seul ou à l'aide d'un cabinet), connaître ses obligations fiscales (calcul de la TVA, des frais déductibles dans le cadre de son activité professionnelle, appels à un conseiller spécialisé, etc.), gérer ses paiements pour éviter les impayés, réclamer les paiements en attente, mettre à jour et archiver les dossiers clients, les bons de commande, les textes cibles, les mémoires de traduction, les glossaires et toutes les autres sources documentaires ;

- le traducteur doit offrir un service de qualité à son client, en d'autres termes apporter les solutions que ce dernier recherche. Et le traducteur économique et commercial doit être capable de s'exécuter rapidement. II doit aussi sans cesse s'efforcer d'améliorer la qualité de ses services en se formant, en corrigeant ses propres erreurs et en travaillant en équipe avec des collègues plus expérimentés capables de réviser ses traductions, etc. Soulignons encore une fois combien il est important que le professionnel sache qu'il ne doit pas offrir des services de traduction dans un domaine qu'il ne maîtrise pas. II vaut mieux refuser un

(11) Selon Ricard Lozano, dans son Vademécum del Traductor. 
travail que livrer une mauvaise traduction. Pour ce qui est de la qualité, force est de constater qu'en Espagne la norme de qualité UNE-EN 15038 (2006) est axée sur la qualité du processus de traduction dans son ensemble, et non sur le produit fini à lui seul ;

- le traducteur doit être à l'écoute du client, l'épauler et lui assurer un service après-vente en cas de problème ou dans l'éventualité d'une erreur de traduction, le but étant d'accompagner et de fidéliser le client ;

- tout comme ses collègues avec lesquels il peut être amené à travailler, le traducteur doit rester motivé, préalable essentiel à son épanouissement professionnel ;

- le traducteur doit être conscient des avantages du travail non salarié, c'est-à-dire l'autonomie, l'indépendance, la flexibilité des horaires et du choix du lieu de résidence par rapport aux inconvénients liés à un flux de travail irrégulier (périodes d'activité intenses et périodes d'inactivité), à l'instabilité des revenus, à un certain isolement dans le travail, etc. ;

- le traducteur doit appartenir à une association. Cet aspect revêt une importance particulière à mes yeux, car, en Espagne, il n'existe pas de Collège officiel de traducteurs et interprètes. Le seul corporatisme est donc celui des associations professionnelles qui offrent une protection et proposent des conseils auprès des traducteurs et des interprètes. Les associations espagnoles comptent davantage de traducteurs indépendants que de traducteurs salariés parmi leurs membres.

Comme il ressort clairement d'une table ronde sur le phénomène associatif dans le secteur de la traduction organisée par l'auteure du présent article à l'Universidad Autónoma de Madrid pour présenter la nouvelle entité VÉRTICE(12), événement qui a réuni des représentants d'ASETRAD, d'ACETT (Sección Autónoma de Traductores de Libros de la Asociación Colegial de Escritores de España) et d'UNICO (Unión de Correctores de España), I'appartenance à une association permet avant tout d'être au courant d'informations relatives à la profession, et offre de nombreux autres avantages tels que la solidarité, l'aide et les conseils face à certains problèmes déontologiques ou professionnels, différentes formations, bourses de travail, publicité via le réseau, contacts avec des collègues. La table ronde a également été l'occasion de constater que le secteur de la traduction est l'un des plus solidaires qui soient. Par exemple, il est assez fréquent qu'un traducteur bénéficie du soutien de collègues plus expérimentés qui travaillent dans le même domaine, une telle solidarité entre collègues étant fondamentale en cas de délais réduits associés à une surcharge de travail.

Si nous faisons le bilan de notre parcours professionnel, l'augmentation de notre clientèle et l'approfondissement de nos connaissances (notamment par le biais des commentaires reçus

(12) Entité appelée à rassembler de nombreuses associations professionnelles du secteur. 
sur nos travaux) tiennent davantage à nos relations avec nos confrères qu'aux relations entretenues avec des clients directs ou des agences de traduction. Notre profession manque encore de maturité et de reconnaissance, c'est pourquoi l'entraide et le travail d'équipe sont si essentiels.

\section{Conclusions}

L'examen de différentes études nationales et internationales sur les perspectives d'avenir du secteur de la traduction permet de conclure qu'il s'agit d'un secteur en croissance. Avec la globalisation et l'internationalisation des entreprises, le recours à la sous-traitance s'impose, plus que jamais, en matière de traduction. Certes, la crise économique mondiale a provoqué quelques restrictions budgétaires, mais l'importance des enjeux financiers oblige les clients à rechercher, avant tout, des traductions de qualité. Le marché de la traduction - marché ouvert non régulé et accessible à tous - est l'un des plus fragmentés qui existe. II se caractérise par une offre atomisée et supérieure à la demande. En revanche, sur le marché de la traduction économique et financière, la concurrence se fait un peu moins féroce. Et l'offre proposée par les traducteurs étant plus restreinte, les tarifs appliqués sont légèrement plus élevés que dans d'autres domaines. Ce phénomène s'explique, d'une part, par une spécialisation plus poussée requise par le traducteur, et, d'autre part, par la complexité et la nature des défis à relever.

Dans cet article, nous avons pu passer en revue certaines particularités du marché de la traduction économique et financière : son hétérogénéité, son dynamisme, son caractère changeant, la difficulté et la complexité de la tâche du traducteur, etc. Les difficultés rencontrées par le traducteur s'expliquent, entre autres, par la diversité des clients et de leurs besoins - auxquels le traducteur doit sans cesse s'adapter -, par la complexité du monde économique actuel, ainsi que par la brièveté des délais impartis.

Sur ce marché exigeant, le traducteur indépendant doit réunir des compétences et des connaissances techniques, documentaires et terminologiques propres à ce vaste domaine de spécialisation. De surcroît, s'il veut obtenir une véritable reconnaissance personnelle et professionnelle, il devra bien maîtriser les outils informatiques de son métier et posséder certaines aptitudes professionnelles.

veronica.roman@uam.es

Article traduit de l'espagnol par Aurélie Barbe. 


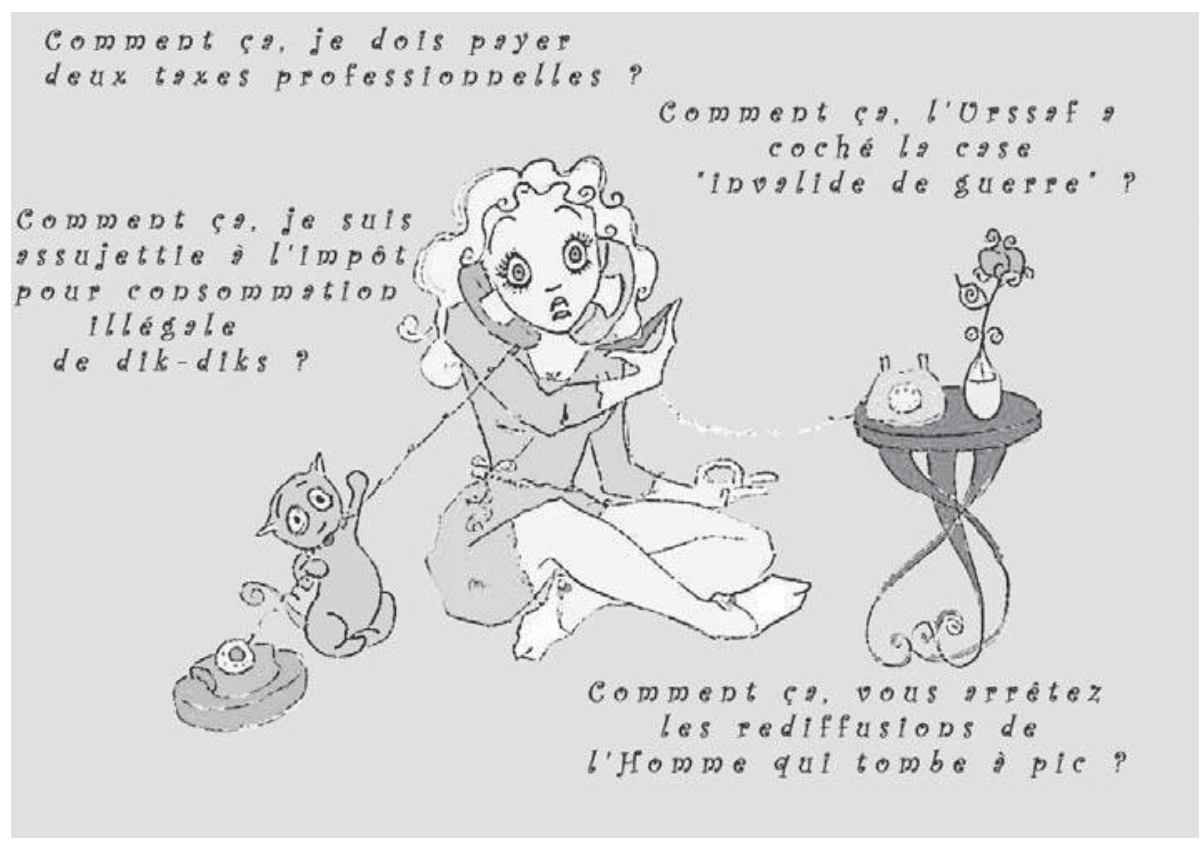

Illustration : Marlène Junius, http://alotoftralala.over-blog.com

\section{Bibliographie}

ACT, (2005). Estudio de situación del mercado español de servicios profesionales de traducción (2004).

AENOR, (2006). Norma Española UNE-EN 15038 Servicios de Traducción. Requisitos para la prestación del servicio. Madrid: AENOR.

AREVALILLO DOVAL, J.J, (2004). " A propósito de la norma europea de calidad para los servicios de traducción ". El español, lengua de traducción I/ Congreso Internacional Toledo. Consultable à l'adresse : http://www.esletra.org/Toledo/html/contribuciones/arevalillo.htm [consulté en janvier 2007].

AREVALILLO DOVAL, J.J, (2006a). " La norma europea de calidad para servicios de traducción EN-15038: por fin una realidad ", Panace@ n. ${ }^{0} 20$. Consultable à l'adresse : http://www.medtrad.org/panacea [consulté en septembre 2011].

AREVALILLO DOVAL, J.J, (2006b). " Visión general de la normalización en el sector de la traducción ». Punto y coma (Boletín de los traductores españoles de las instituciones de la Unión 
Être traducteur économique et financier aujourd'hui : défis et avantages

Europea, $\mathrm{n}^{0}$ 100. Consultable à l'adresse : http://ec.europa.eu/translation/bulletins/puntoycoma/100/pyc1005 es.htm [consulté en mai 2010].

COMMON SENSE ADVISORY, INC., (2010). Language Services Market 2010. Consultable à l'adresse : http://www.commonsenseadvisory.com [consulté en octobre 2011].

EUATC (European Union of Associations of Translation Companies), (2005). The European Translation Industry: Facing the Future.

HAGER, A.,(2008). "The translation market in ten years' time - a forecast ". Consultable à l'adresse : http://www.tcworld.info/tcworld/translation-and-localization/article/the-translationmarket-in-ten-years-time-a-forecast/ [consulté en septembre 2011].

LOZANO, R., Vademecum del traductor. Página web del traductor autónomo Ricard Lozano. Consultable à l'adresse : http://www.rlozano.com/ [consulté en novembre 2007].

MAYOR SERRANO, AREVALILLO, CASTRO ROIG, DE MIGUEL y TURRIÓN, (2004). "Salidas profesionales del traductor médico ". Consultable à l'adresse : www.medtrad.org/panacea.htm Panacea. Volumen V n ${ }^{0} 16$. [consulté en juin 2005].

MICROSOFT PYMES Y AUTÓNOMOS. Traducir para exportar. Consultable à l'adresse : http://www.microsoft.com/business/es-es/Content/Paginas/article.aspx?cbcid=224.

[consulté en novembre 2011].

\section{Articles de presse et sites web intéressants sur Internet}

ACT, www.act.es

AENOR, www.aenor.es

ASETRAD, http://www.asetrad.org/

CEN, www.cenorm.be

El País, http://www.elpais.com/articulo/economia/global/exportaciones/rescate/Espana/elpepueconeg/20110227elpnegeco 1/Tes

El Plural, http://www.elplural.com/economia/el-crecimiento-de-las-exportaciones-coloca-aespana-en-el-quinto-puesto-del-ranking-mundial/

EUATC, www.euatc.org

Mercado de trabajo, "intérpretes de la palabra " del 16 al 22 de noviembre de 2007. www2.uah.es/traduccion/novedades/EspecialTraduccion.pdf. [consulté en octobre 2011] 
Verónica Román

New York Times,

http://www.nytimes.com/2006/04/30/nyregion/30homefront.html?ex $=1304049600$ \&en $=5 \mathrm{ced}$ 97b426f03864\&ei=5090\&partner=rssuserland\&emc $=r s s$

UNICO, http://www.uniondecorrectores.org/

VÉRTICE, http:asocesp.blogspot.com

Verónica Román est diplômée en droit de l'Université de Valladolid (1994) et en traduction et interprétation du CES Felipe II de Aranjuez (Universidad Complutense de Madrid, 2004). Elle est traductriceinterprète assermentée pour la langue anglaise par le ministère des Affaires étrangères en Espagne et a soutenu une thèse de doctorat en traduction et interprétation auprès de l'Université de Málaga. Elle se consacre à la traduction professionnelle depuis 1996. Après avoir fait ses premières armes en tant que traductrice-interprète salariée dans différentes multinationales, elle a été associée de l'entreprise Traducciones.com. Verónica Román est actuellement à son compte en tant que traductrice indépendante assermentée (anglais-espagnol) et correctrice de textes juridiques et économiques, activité qu'elle partage avec l'enseignement de la traduction en France (ISIT) et en Espagne (Université de Castilla-La Mancha, Université autonome de Madrid). 